\title{
Problemas de saúde mental na adolescência: características familiares, eventos traumáticos e violência
}

\author{
Silvia Pereira da Cruz Benetti ${ }^{-}$- Universidade do Vale do Rio dos Sinos, São Leopoldo, Brasil \\ Adriana Pizetta - Universidade do Vale do Rio dos Sinos, São Leopoldo, Brasil \\ Cristian Baqui Schwartz - Universidade do Vale do Rio dos Sinos, São Leopoldo, Brasil \\ Raíssa de Azevedo Hass - Universidade do Vale do Rio dos Sinos, São Leopoldo, Brasil \\ Vera Lúcia Melo - Universidade do Vale do Rio dos Sinos, São Leopoldo, Brasil
}

\begin{abstract}
Resumo
A ocorrência de situações traumáticas, exposição à violência e características das relações parentais são fatores de risco para saúde mental. Este estudo procurou identificar as situações individuais, familiares e contextuais associadas às manifestações de problemas de saúde mental em adolescentes estudantes da região metropolitana de Porto Alegre, RS. Os instrumentos utilizados foram o Inventário de Eventos Estressores na Adolescência, Triagem da Exposição de Crianças à Violência na Comunidade, Inventário de Estilos Parentais e o Child Behavior Checklist- versão YRF. Participaram 245 adolescentes, sendo 114 (46,5\%) do sexo masculino com idade média de 16,02 anos ( $\mathrm{DP}=1,83)$. A análise dos dados realizou-se através de estatística descritiva e inferencial para estimar os determinantes do diagnóstico clínico. Situações de violência, práticas parentais negativas e eventos traumáticos associaram-se ao diagnóstico clínico. Aponta-se a necessidade de implantação de ações preventivas e de atenção em saúde mental na infância e adolescência.

Palavras-chave: Adolescência, Perturbação emocional, Relações familiares, Eventos estressantes.
\end{abstract}

\section{Mental health problems in adolescence: family characteristics, traumatic events and violence}

\begin{abstract}
The occurrence of traumatic events, exposure to violence and characteristics of parental relationship are risk factors associated to mental health. This study aimed to identify individual, family and contextual factors associated to the manifestation of mental health problems in a sample of adolescent students from the metropolitan region of the city of Porto Alegre, RS. The instruments used were the Stressful Adolescence Events Inventory, Screening of Exposure to Community Violence, Parental Style Inventory and the Child Behavior Checklist- YRF version. Two hundred and forty five adolescents, of which $114(46,5 \%)$ male, mean age 16,02 (DP=1,83) participated in the study. Data analysis was done through descriptive and inferential statistics to estimate the determinants of clinical diagnostics. Violence situations, negative parenting practices and traumatic events were associated to clinical diagnosis. It is appointed the need to implement preventive actions and intervention geared to child and adolescent mental health.
\end{abstract}

Keywords: Adolescence, Emotional disturbance, Family relations, Stressful events.

Estima-se que, no nível mundial, segundo dados da Organização Mundial de Saúde (OMS, 2003), cerca de $20 \%$ de crianças e adolescentes apresentam algum problema de saúde mental, situação que pode trazer consequências negativas a curto e longo prazo nas trajetórias de desenvolvimento dos indivíduos. No Brasil, o "Fórum Nacional de Saúde Mental InfantoJuvenil" identificou como prioritárias ações voltadas para a prevenção e reversão da institucionalização de crianças portadoras de transtornos psiquiátricos. Portanto, para a efetivação dessas ações, o incremento de estudos sobre a saúde mental na infância e adolescência constitui-se como uma diretriz fundamental tanto dirigida para a prevenção como para a implementação de programas de atendimento.

\footnotetext{
${ }^{1}$ Endereço para correspondência

Rua Riveira, 150/301 - 90670-160 - Porto Alegre, RS

E-mail: sbenetti@unisinos.br

Apoio CNPq/FAPERGS/UNISINOS
}

Um campo importante de estudos sobre situações de maior vulnerabilidade para a manifestação de transtornos mentais é a área da Psicopatologia do Desenvolvimento (Bastos, Urpia, Pinho \& Almeida Filho, 1999; Rutter, 2009). Basicamente, as pesquisas nesse campo fundamentam-se no modelo multidimensional de etiologia dos transtornos mentais, considerando uma perspectiva dinâmica e transacional entre os diferentes sistemas individuais, familiares e sociais. Além disto, o modelo compreende a interação desses elementos de forma processual, ao longo do desenvolvimento individual, tendo maior impacto em momentos evolutivos marcados por maior transição, tal como as transformações na puberdade e início da vida adulta (Cicchetti \& Rogosh, 2002, Shirk, Talmi, Olds, 2000).

As áreas de maiores dificuldades na adolescência incluem as oscilações de humor, o engajamento em comportamentos de risco e os conflitos intensos nas relações familiares (Cicchetti \& Rogosh, 2002). Pesquisas epidemiológicas e clínicas têm utilizado duas dimensões psicopatológicas como 
base para a identificação da presença de transtornos. Uma delas, internalização, refere-se às manifestações de sintomas de ansiedade, depressão, somáticos e obsessivos, caracterizados fundamentalmente por aspectos internos. Já a dimensão externalização corresponde aos comportamentos agressivos, uso de substâncias, conduta antissocial e delinquência (Assis, Avanci, Pesce, \& Ximenes, 2009; Carvalho, Pinheiro, Patrick, Krueger \& Markon, 2007). Por sua vez, a manifestação desses quadros clínicos tem sido associada a determinadas características individuais, familiares, contextuais e experiências de eventos traumáticos (Benvegnu, Fassa, Facchini, Wegman \& Dall' Agnol, 2005; Paula, Duarte \& Bordin, 2007; Tick, Ende \& Verhulst, 2008).

A ocorrência de eventos traumáticos ou estressores é comum durante o desenvolvimento humano, porém, o efeito cumulativo ao longo da vida de situações traumáticas intensifica as chances de manifestação de psicopatologia (Dekovic, 1999). Experiências de violência, condições de pobreza, rompimentos de vínculos familiares, mortes e doenças crônicas são algumas das situações caracterizadas como fatores de risco crônicos ou agudos para a ocorrência de transtornos mentais, principalmente considerando a intensidade e frequência (Pesce, Assis, Santos \& Oliveira, 2004). Em relação à adolescência, pesquisas internacionais (Waaktar, Borge, Fundingsrud, Christie \& Torgesen, 2004) e nacionais (Wathier \& Dell' Áglio, 2007) apontam que problemas de internalização associam-se a situações traumáticas de perdas e separações das figuras parentais. Por exemplo, Wathier e Dell'Áglio, numa investigação com 257 jovens na região metropolitana de Porto Alegre, dos quais 130 residiam em abrigos de proteção, identificaram que a maior frequência de eventos estressores associava-se à manifestação de sintomas depressivos. O grupo de jovens em situação de acolhimento e, portanto, vivenciando rompimento de laços familiares, apresentou maior incidência de sintomas depressivos. Em acréscimo, King e Chassin (2008) apontam que os eventos traumáticos não somente intensificam as manifestações depressivas e de comportamento agressivo na adolescência, como também predispõem para o abuso de substâncias.

Por sua vez, problemas familiares e extrafamiliares, e violência em geral (Dekovic, 1999, Waaktar \& cols., 2004) associam-se às manifestações externalizantes, ou seja, o envolvimento do adolescente com ações agressivas e delinquentes. $\mathrm{Na}$ esfera relacional, portanto, a família surge como um fator de promoção da saúde psicológica, principalmente considerando-se a importância da qualidade das relações familiares vinculadas aos estilos parentais
(Gomide, Salvo, Pinheiro \& Mello, 2005).

Esses estilos consistem no contexto emocional das relações entre pais e filhos, no qual as práticas parentais mais específicas são implementadas, principalmente em relação ao cuidado e supervisão dos filhos (Darling \& Steinberg, 1993). Essas práticas de socialização organizam-se em torno das dimensões de apoio emocional, afeto e exigência, entendida como a capacidade dos pais de oferecer suporte emocional e preocupação com o bem-estar da criança, ao mesmo tempo em que exibem controle e supervisão do comportamento, e das dimensões de hostilidade/coerção, as quais se organizam pelos comportamentos coercitivos, manipulativos e ausência de limites (Lovejoy, Weis, O’Hare \& Rubin, 1999). Dessa maneira, práticas parentais caracterizadas por atitudes de negligência, omissão, afetos negativo ou punição excessiva estão associadas à manifestação de transtornos emocionais (Carvalho \& Gomide, 2005, Galambos, Barker \& Almeida, 2003; Gomide, Salvo, Pinheiro \& Mello, 2005). Por exemplo, em um estudo com 48 adolescentes brasileiros divididos em dois grupos, sendo um deles de 24 jovens com diagnóstico de dependência química, Broecker e Jou (2007) identificaram que práticas parentais socialmente indesejáveis estavam presentes no grupo de dependentes. Nesse grupo, a interação com os pais foi descrita como baseada na intromissão, no controle culposo, na autoridade, nas dificuldades de relacionamento e na inconsistência na disciplina.

No Brasil, Gomide (2006), com base nas práticas parentais e estratégias de socialização de crianças e adolescentes, considera que o estilo parental, ou seja, o conjunto de práticas, valores e atitudes utilizadas pelos pais para socializar os filhos, inclui práticas parentais positivas e práticas negativas. As primeiras são orientadas para o comportamento pró-social, tais como comportamento moral e monitoria positiva. Já as práticas parentais negativas são associadas ao comportamento antissocial, incluindo negligência, punição inconsistente, disciplina relaxada, monitoria negativa e abuso físico. Considerando esses aspectos, Carvalho e Gomide (2005) identificaram em 41 famílias de jovens envolvidos com a lei que o estilo parental materno e paterno foi percebido pelos jovens como de risco. Isto é, prevaleceram práticas associadas à negligência, disciplina inconsistente, monitoria negativa e abuso físico em ambos os pais, indicando dificuldades importantes na esfera familiar no tocante à socialização dos filhos. Ainda, nesse estudo, tanto os pais como os filhos responderam ao inventário, tendo sido identificadas similaridades entre as avaliações realizadas no nível parental e no nível dos jovens. Em termos de pesquisa, esse resultado reforça a validade de 
investigações que se utilizam de dados obtidos fundamentando-se na percepção dos próprios jovens.

Por sua vez, Proust e Gomide (2007) focalizaram-se na identificação de características de comportamento moral entre famílias de risco e de nãorisco, avaliadas por meio do Inventário de Estilo Parental (Gomide, 2006). Cada grupo foi composto por 30 famílias, incluindo os pais e os filhos adolescentes. Os resultados apontaram que as familias de não-risco apresentaram um maior nível de comportamento moral do que as famílias de risco. A importância da dimensão comportamento moral relaciona-se ao aspecto de que essa prática educativa refere-se justamente à transmissão dos valores e normas sociais que os pais possibilitam à criança e ao adolescente mediante interações afetivas e de caráter corretivo e de responsabilidade pelos atos. Portanto, famílias de risco apresentam dificuldades no envolvimento com práticas afetivas, empáticas e vinculadas ao ensino de valores e ideais.

Finalmente, estudos com jovens provenientes de regiões metropolitanas de grandes centros urbanos indicam que a exposição à violência tem aumentado nessas faixas etárias (Avanci, Assis, Oliveira \& Pires, 2009; Benetti \& cols., 2006; Zavaschi, Benetti \& Polanczyk, 2002). Nesse sentido, este aumento de atos violentos nas comunidades foi considerado como uma situação epidêmica em saúde pública (Waiselfisz, 2000). Assim, em função da intensidade e frequência de episódios violentos envolvendo agressões, estupros, assaltos, roubos, tráfico de drogas, determinadas regiões urbanas caracterizam-se como em situação de violência comunitária crônica, sendo as crianças e os adolescentes geralmente os mais expostos ou sofrendo vitimização por esses atos (Osofsky, 1995).

As investigações sobre a exposição à violência comunitária indicam que seus efeitos podem se manifestar a curto e longo prazo (Margolin \& Gordis, 2004), incluindo alterações fisiológicas e psicológicas. Crianças e adolescentes vítimas diretas e indiretas da violência comunitária manifestam primeiramente distúrbios do comportamento, agressividade, distúrbios do humor, e sintomas de estresse pós-traumático, como pesadelos, flashbacks, problemas clínicos de saúde e dificuldades escolares relacionadas ao desempenho cognitivo (Osofsky, 1995; McFarland \& cols., 2003; Rosenthal \& Wilson, 2003). Em longo prazo, a exposição à violência foi associada ao comportamento agressivo na adolescência, envolvimento com drogas e a polícia, e depressão (Paxton, Robinson, Shah \& Schoeny, 2004). Finalmente, estudos com amostragens clínicas têm encontrado também que adolescentes de risco (presença de condutas antissociais e/ou sintomas depressivos) foram expostos a um maior número de incidentes de violência e vitimização do que grupos controle (Flannery, Singer \& Wester, 2001).

Considerando esses aspectos, este trabalho tem como objetivo identificar as situações individuais, familiares e contextuais associadas às manifestações de desordens emocionais em adolescentes estudantes do ensino médio da região metropolitana de Porto Alegre. Especificamente, por meio do instrumento CBCLversão YRF, um inventário utilizado para o rastreamento de problemas de saúde mental em adolescentes (Assis, Avanci, Pesce \& Ximenes, 2009; Bordin \& cols., 2009), procurou-se verificar a prevalência e os fatores associados às manifestações clínicas de problemas de internalização (retraimento, queixas somáticas, ansiedade/depressão) e de externalização (comportamento de quebrar regras e comportamento agressivo).

\section{Método}

Trata-se de um estudo descritivo, quantitativo e transversal, que procurou identificar as características dos eventos de vida traumáticos e redes de apoio de adolescentes da região metropolitana de Porto Alegre.

\section{Participantes}

Participaram desta pesquisa 245 adolescentes, dos quais $114(46,5 \%)$ do sexo masculino e 131 $(53,5 \%)$ do sexo feminino. Este número foi calculado considerando-se os valores de exposição (diagnóstico clínico de 20\%), utilizando-se do programa Epi-Info 6.0 , poder de $80 \%$ e nível de significância de $p=0,05$. A idade média dos jovens foi de 16,02 anos (DP = 1,83). Em relação à escolaridade, 81 adolescentes $(33,1 \%)$ estavam no ensino fundamental e $164(66,9 \%)$ no ensino médio, sendo que $52,8 \%$ das meninas e $71,8 \%$ dos meninos cursavam séries atrasadas em relação à idade correspondente, apresentando uma defasagem de dois anos em sua escolaridade ( $M=23,01$ meses, $\mathrm{DP}=21,27)$. Quanto aos arranjos familiares, 108 adolescentes residiam com o pai e a mãe (46,4\%), 63 somente com a mãe $(27,0 \%), 11$ apenas com o pai (4,7\%), 15 com o pai ou mãe e madrasta ou padrasto $(6,4 \%)$ e $35(15 \%)$ em outras composições familiares.

\section{Instrumentos}

Inventário de Estilos Parentais (IEP)

O Inventário de Estilos Parentais (Gomide, 2006) consiste de 42 itens referentes a sete práticas educativas parentais que caracterizam o modo como os pais se relacionam atualmente com os seus filhos. As práticas parentais positivas incluem a monitoria positiva (conhecimento de onde o filho se encontra, de suas atividades, gostos e preferências, demonstração de 
afeto) e comportamento moral (transmissão de valores como honestidade, generosidade, senso de justiça, discriminação do certo e do errado). As práticas parentais negativas incluem a punição inconsistente (disciplina baseada no estado afetivo parental), negligência (não-atenção às necessidades infantis, ausência de responsabilidade, auxílio e afeto), disciplina relaxada (não-cumprimento de regras), monitoria negativa (excesso de fiscalização, rigidez) e abuso físico (punição corporal excessiva). Calcula-se o índice de Estilo Parental (IEP) pela soma das práticas positivas da qual é subtraída a soma das práticas negativas. Os resultados variam de +24 (estilo parental excelente) a 60 (estilo parental de extremo risco). Portanto, o índice negativo indica a presença de práticas educativas negativas e, ao contrário, índices positivos apontam para práticas positivas no processo de socialização. $\mathrm{O}$ alfa de Cronbach do Inventário referente às práticas paternas foi igual a 0,79 e da materna igual a 0,92 , sendo a consistência interna total do instrumento igual a 0,86 .

\section{Inventário de Eventos Estressores na Adolescência (IEEA)}

Os eventos estressores foram avaliados pelo Inventário de Eventos Estressores na Adolescência (Ferlin, Lima, Alchieri, Kristensen \& Flores, 2000). O instrumento é composto por 64 itens na forma de eventos de vida estressores. Para cada item, o participante deve indicar, em uma alternativa sim/não, se o evento ocorreu e, a partir disso, em uma escala Likert de cinco pontos, informar o impacto atribuído a cada evento. Neste trabalho foram avaliados os escores referentes ao número total de eventos (ET), a média de eventos (ME) e intensidade (IE). Além disso, foram calculadas as médias por dimensões de eventos. Essas dimensões foram organizadas em estudo anterior (Dell'Aglio \& cols., 2005) considerando os domínios dos eventos e envolvendo diretamente o jovem ou as pessoas naquele contexto: Dominio Familiar (DF)- 17 situações envolvendo conflitos diretamente ligados ao ambiente familiar (p. ex., morte de outro familiar, ter que obedecer às ordens dos pais, ter brigas com irmãos, um dos pais ter filhos com outros parceiros, um dos pais ficar desempregado); Dominio Escolar (DE)9 situações de eventos ocorridos na escola (p. ex., ter provas no colégio, mudar de colégio, rodar de ano na escola, ser expulso(a) da sala de aula pela professora, ter problemas com professores, ser suspensa da escola); Dominio Social (DS)- 15 eventos associados ao relacionamento do jovem com os demais (p. ex., ser xingada ou ameaçada verbalmente, terminar o namoro, discutir com amigos, mudar de casa ou de cidade, envolver-se em brigas com agressão física); Dominio Judicial (DJ)- eventos envolvendo esferas legais, conselho tutelar, abrigos (ser levada para FASE, problemas com a justiça, problemas com a polícia, ir para o Conselho Tutelar, ser levada para uma instituição de abrigo; Domínio Sexual (DSE)- 4 situações envolvendo questões de sexualidade (ficar grávida, ser tocada sexualmente contra a vontade, fazer aborto, ser estuprada); e Dominio Pessoal (DP)- 14 situações de vida não caracterizadas anteriormente (p. ex., ter crise nervosa, usar drogas, ter dúvidas quanto à aparência e mudanças no corpo, ter sofrido algum tipo de violência, sofrer acidente, ter dormido na rua). No presente estudo, o instrumento apresentou um alfa de Cronbach igual a 0,95 .

\section{Questionário Triagem da Exposição de Crianças à Violência na Comunidade}

Este questionário, elaborado para utilização em distintas comunidades para a identificação de eventos violentos pelo National Institute of Mental Health - EUA (Richters \& Martinez, 1993) e utilizado no contexto nacional (Zavaschi \& cols., 2002), consiste em 49 questões auto-respondíveis abordando quatro tipos de violência: Violência Comunitária, Familiar, Sexual e Exposição às Drogas, das quais os adolescentes possam ter sido as próprias vítimas (Exposição Direta), possam ter presenciado como testemunhas, ou possam ter ouvido falar sobre tais situações (Exposição Indireta) e uma questão aberta para que situações não-listadas sejam identificadas (Zavaschi \& cols., 2002). Neste estudo, o instrumento apresentou um alfa de Cronbach igual a 0,89 .

\section{Child Behavior Checklist (CBCL)}

O CBCL é um Inventário de Comportamentos na Infância e Adolescência (Achenbach, 1991, Bordin, Mari \& Caeiro, 1995). Trata-se de um instrumento validado internacionalmente e utilizado para investigar problemas de saúde mental na infância e adolescência. A primeira parte avalia a Competência Social e é constituída por três escalas individuais: Atividades, Sociabilidade e Escolaridade, cuja soma dá origem à Escala Total de Competência Social (CS). A segunda parte, Problemas de Comportamento, possui oito escalas de síndromes (Retraimento, Queixas Somáticas, Ansiedade/Depressão, Problemas Sociais, Problemas do Pensamento, Problemas de Atenção, Problemas Sexuais, Comportamento de Quebrar Regras e Comportamento Agressivo). As três primeiras subescalas correspondem à escala de comportamentos do tipo internalização, enquanto as duas últimas subescalas correspondem à escala de comportamentos do tipo externalização. Para a realização dessa pesquisa foi utilizada a versão Y. R. F (Questionário de autoavaliação para jovens - 11 a 18 anos) do CBCL, na qual 
o próprio adolescente responde o questionário. A soma das escalas permite a classificação dos valores mediante escores padronizados em categoria não-clínica - abaixo de 63 pontos, categoria limítrofe de 64 e categoria clínica - acima de 65 pontos. Portanto, a soma de todas as subescalas gera o escore total de problemas de saúde mental. No presente trabalho, utilizamos o ponto de corte no valor de escore de 65 pontos.

\section{Procedimentos}

Para realização do estudo transversal, inicialmente, foram contatadas a Secretaria Municipal de Educação do Município de São Leopoldo e a Secretaria Estadual de Educação e Cultura do Rio Grande do Sul para obter-se a autorização para a realização da pesquisa. A coleta de dados foi realizada em três escolas públicas por auxiliares de pesquisa que foram devidamente treinados para a aplicação dos instrumentos. Essas escolas foram selecionadas por situarem-se na região central da cidade, portanto, acolhendo alunos de todos os bairros do município. Esta característica oferece maior heterogeneidade à amostra. Além disso, foi solicitado o consentimento dos pais ou responsável pelo adolescente, obtido mediante a assinatura do Termo de Consentimento Livre e Esclarecido (TCLE) elaborado conforme as diretrizes regulamentadoras de pesquisa envolvendo seres humanos, Resolução no 196/96 do CNS, e aprovado pelo Comitê de Ética da UNISINOS. Nos casos de identificação de situação que envolvesse dano, risco ou demanda de atendimento psicológico, os adolescentes foram encaminhados para a clínica-escola da universidade.

\section{Análise dos dados}

A análise dos dados foi feita por estatística descritiva, frequência e percentual, e inferencial, regressão linear método stepwise, seguidas do teste de ANOVA, para estimar as variáveis mais importantes na determinação do diagnóstico clínico, adotando o nível de significância de 0,05 .

\section{Resultados}

A avaliação do CBCL indicou que dos 245 adolescentes investigados neste estudo, 169 não apresentaram escore clínico (69\%), 34 (13,9\%) apresentaram diagnóstico clínico de internalização e 42 $(17,1 \%)$ de externalização. Dentre o grupo de jovens que obtiveram escore clínico internalização, 15 eram do sexo masculino e 19 do feminino. No grupo de externalização, 14 eram do sexo masculino e 28 do feminino. As médias das escalas internas do CBCL apontaram diferenças significativas na presença de problemas somáticos quanto ao gênero (masculino $\mathrm{M}=55,37$, $\mathrm{DP}=6,62$ e feminino $\mathrm{M}=57,33, \mathrm{DP}=7,07, \mathrm{p} 0,02)$; em problemas de atenção (masculina $\mathrm{M}=54,86, \mathrm{DP}=6,71 \mathrm{e}$ feminina $\mathrm{M}=57,50, \quad \mathrm{DP}=7,41, \quad \mathrm{p}<01) ; \mathrm{e} \quad \mathrm{em}$ comportamento agressivo (masculino $\mathrm{M}=55,53, \mathrm{DP}=6,58 \mathrm{e}$ feminino $\mathrm{M}=58,84, \mathrm{DP}=8,37, \mathrm{p}<01)$.

Em relação às práticas educativas parentais avaliadas por meio do Inventário de Estilos Parentais (IEP), verificou-se que 44,7\% $(n=89)$ dos adolescentes classificaram as Práticas Parentais Paternas no repertório de risco, 30,2\% ( $\mathrm{n}=60)$ bom, abaixo da média, $14,1 \% \quad(\mathrm{n}=28)$ bom, acima da média e $11 \%$ $(\mathrm{n}=22)$ ótimo. Quanto ao Estilo Parental Materno (EPM), os resultados apontaram que 38,9\% ( $\mathrm{n}=86)$ foram classificados como de risco, 30,3\% $(n=67)$ bom, abaixo da média, 20,8\% ( $\mathrm{n}=46)$ bom, acima da média e $10 \% \quad(n=22)$ ótimo. Nesse sentido, tanto o Índice Parental Paterno $(M=-1,76, D P=9,11)$ quanto o Índice Parental Materno $(\mathrm{M}=-0,27, \quad \mathrm{DP}=8,30)$ foram negativos, classificados na faixa de risco.

A análise específica das práticas educativas paternas indica que as médias das práticas positivas de monitoria positiva $\mathrm{M}=6,29 \quad(\mathrm{DP}=3,26)$ e as de comportamento moral $\mathrm{M}=6,81$ ( $\mathrm{DP}=3,15)$ foram baixas, caindo na faixa de risco. Já as práticas negativas abuso físico $\mathrm{M}=0,95(\mathrm{DP}=1,55)$ caíram na faixa estilo parental bom; negligência $(\mathrm{DP}=2,59)$ bom, porém abaixo da média; monitoria negativa $\mathrm{M}=4,16 \quad(\mathrm{DP}=2,44)$ bom; punição inconsistente $\mathrm{M}=3,18(\mathrm{DP}=2,29)$ bom; e disciplina relaxada $\mathrm{M}=2,94$ ( $\mathrm{DP}=2,42)$ bom, acima da média.

Quanto ao estilo materno, as médias das práticas educativas positivas monitoria positiva $\mathrm{M}=8,05$ $(\mathrm{DP}=2,75)$, como sendo de risco; comportamento moral $\mathrm{M}=7,63$ ( $\mathrm{DP}=2,81)$, os índices são baixos, classificados como de risco; abuso físico $\mathrm{M}=0,93$ ( $\mathrm{DP}=1,58)$, bom; negligência $\mathrm{M}=2,75(\mathrm{DP}=2,28)$, bom; monitoria negativa $\mathrm{M}=5,47$ ( $\mathrm{DP}=2,54)$, bom; punição inconsistente $\mathrm{M}=3,50$ $(\mathrm{DP}=2,21)$, bom; e disciplina relaxada $\mathrm{M}=3,29$ $(\mathrm{DP}=2,27)$, bom, porém abaixo da média.

A média geral da ocorrência dos eventos estressores foi semelhante para ambos os sexos: feminino $(\mathrm{M}=17,00, \quad \mathrm{DP}=10,05)$ e masculino $(\mathrm{M}=15,83$, $\mathrm{DP}=9,87)$. Igualmente, não houve diferenças em relação ao impacto geral dos eventos. Os jovens do sexo masculino tiveram média de $\mathrm{M}=37.62$ ( $\mathrm{DP}=34,76)$ e feminino $\mathrm{M}=45,41 \quad(\mathrm{DP}=38,58)$. As situações estressoras mais frequentes foram: Ter que obedecer as ordens de seus pais $(\mathrm{n}=146,73,0 \%)$, Discutir com os amigos $(\mathrm{n}=150,75,8 \%)$, Brigas com irmãos $(\mathrm{n}=127$, 65,5\%) e Terminar o namoro ( $\mathrm{n}=109,45,6 \%$ ). Em relação à intensidade, os eventos de maior impacto foram: Morte de irmãos $(\mathrm{M}=3,78 ; \mathrm{DP}=1,62)$, Ter que viver em abrigo $(\mathrm{M}=3,80 ; \mathrm{DP}=0,91)$, Ser impedido de ver os pais $(\mathrm{M}=4,00 ; \mathrm{DP}=1,41)$, Morte de um dos pais $(\mathrm{M}=4,06$; 
$\mathrm{DP}=1,49)$, Ser estuprado $(\mathrm{M}=4,20 ; \mathrm{DP}=1,47)$. As médias das dimensões foram: Domínio Escolar (DE) $(\mathrm{M}=3,17$; $\mathrm{DP}=1,73)$, Judicial (DJ) $(\mathrm{M}=1,08, \mathrm{DP}=0,89)$, Sexual (DS) $(\mathrm{M}=0,44, \mathrm{DP}=0,86)$, Pessoal (DP) $(\mathrm{M}=3,68$; $\mathrm{DP}=2,60)$, Social (DS) $(\mathrm{M}=3,73$; $\mathrm{DP}=2,86)$ e Familiar (DF) $(\mathrm{M}=4,65 ; \mathrm{DP}=2,90)$. O teste $t$ de Student indicou que a média da dimensão familiar foi significativamente mais alta que as demais $(t=-5,99, p=0,00)$. As dimensões pessoal e social tiveram médias similares $(\mathrm{t}=0,380, \mathrm{p}=0,70)$.

Quanto à exposição aos Eventos de Violência (EVI), identificou-se uma média de 68,75 (DP=10,19) eventos. No geral, a média de exposição masculina total $(\mathrm{M}=69,76, \mathrm{DP}=12,03)$ foi maior que a feminina $(\mathrm{M}=67,83, \mathrm{DP}=8,13, \mathrm{p}<05)$, bem como na dimensão de exposição às drogas (masculino $\mathrm{M}=6,50, \mathrm{DP}=1,35$; feminino $\mathrm{M}=5,93, \quad \mathrm{DP}=1,38 ; \mathrm{p}<01)$. A violência comunitária apresentou uma média elevada para ambos os sexos (masculino $\mathrm{M}=51,62, \mathrm{DP}=9,28$, feminino $\mathrm{M}=50,15, \mathrm{DP}=6,03)$. Em relação à exposição direta a eventos violentos, a média foi de $\mathrm{M}=15,90(\mathrm{DP}=2,28)$ e a exposição indireta $\mathrm{M}=52,68$ ( $\mathrm{DP}=8,50)$. Os resultados de médias e desvio padrão de Diagnóstico Clínico (CBCL), Eventos Estressores (EEV) e Exposição à Violência (EVI) estão descritos na Tabela 1.

Tabela 1 - Médias de diagnóstico clínico, eventos estressores e exposição à violência por sexo dos adolescentes

\begin{tabular}{|c|c|c|c|}
\hline \multirow[b]{2}{*}{ Diagnóstico Clínico (CBCL) } & \multicolumn{2}{|c|}{ Sexo } & \multirow[t]{2}{*}{ Total } \\
\hline & $\begin{array}{l}\text { Masculino } \\
\text { M (DP) }\end{array}$ & $\begin{array}{c}\text { Feminino } \\
\text { M (DP) }\end{array}$ & \\
\hline Internalização & $55,54(8,96)^{*}$ & $57,82(8,92)^{*}$ & $56,73(8,99)$ \\
\hline Externalização & $54,78(8,74)^{* * *}$ & $58,2010,06)^{* * *}$ & $56,57(9,58)$ \\
\hline Escore Clínico Total & $55,468,22)^{* *}$ & $58,14(8,96)^{* *}$ & $56,86(8,70)$ \\
\hline \multicolumn{4}{|l|}{ Eventos Estressores (EVE) } \\
\hline Domínio Familiar & $4,30(3,00)$ & $4,92(2,80)$ & $4,65(2,90)$ \\
\hline Domínio Escolar & $3,34(1,73)$ & $3,04(1,73)$ & $3,17(1,73)$ \\
\hline Domínio Social & $3,63(2,80)$ & $3,81(2,93)$ & $3,73(2,86)$ \\
\hline Domínio Judicial & $1,04(0,87)$ & $1,11(0,92)$ & $1,08(0,89)$ \\
\hline Domínio Sexual & $0,44(0,85)$ & $0,44(0,87)$ & $0,44(0,86)$ \\
\hline Domínio Pessoal & $3,34(2,56)$ & $3,94(2,61)$ & $3,68(2,60)$ \\
\hline Eventos Estressores Total & $15,83(9,87)$ & $17,00(10,05)$ & $16,49(9,96)$ \\
\hline \multicolumn{4}{|l|}{ Exposição à Violência (EVI) } \\
\hline Violência Comunitária & $51,62(9,28)$ & $50,15(6,03)$ & $50,85(7,76)$ \\
\hline Violência Familiar & $4,02(1,08)$ & $4,20(1,05)$ & $4,11(1,06)$ \\
\hline Violência Sexual & $3,34(0,65)$ & $3,34(0,56)$ & $3,34(0,60)$ \\
\hline Violência Jurídica & $4,61(0,92)$ & $4,26(0,80)$ & $4,43(0,87)$ \\
\hline Violência de Exposição às Drogas & $6,50(1,35)$ & $5,93(1,38)$ & $6,20(1,40)$ \\
\hline Violência Total & $69,76(12,03)^{*}$ & $67,83(8,13)^{*}$ & $68,75(10,19)$ \\
\hline
\end{tabular}

${ }^{*} \mathrm{p}<0,05{ }^{* *} \mathrm{p}<0,03{ }^{* * *} \mathrm{p}<0,01$

Com objetivo de verificar as associações entre as manifestações das desordens emocionais, o Escore Clínico Total (ECT) obtido pela avaliação do CBCL foi correlacionado com as variáveis investigadas. Identificou-se que o ECT apresentou correlação positiva com a média total de exposição aos Eventos de Violência $(\mathrm{r}=0,26, \mathrm{p}<01)$, e com as dimensões violência familiar $(\mathrm{r}=0,23, \mathrm{p}<01)$; violência comunitária $(\mathrm{r}=0,26$, $\mathrm{p}<01)$; violência direta $(\mathrm{r}=0,24, \mathrm{p}<01)$; violência indireta $(\mathrm{r}=0,24, \mathrm{p}<01)$ e exposição a drogas $(\mathrm{r}=0,15, \mathrm{p}<02)$. Também foram identificadas correlações negativas entre o ECT e o Estilo Parental Paterno (EPP) ( $r=$ 0,313, $\mathrm{p}=0,00)$, assim como o Estilo Parental Materno $(\mathrm{EPM})(\mathrm{r}=-0,20, \mathrm{p}=03)$ e as práticas monitoria positiva paterna $(\mathrm{r}=-0,15, \mathrm{p}=03)$. O Escore Clínico Total (ECT) correlacionou-se positivamente com punição materna $(\mathrm{r}=0,18, \mathrm{p}<01)$, negligência materna $(\mathrm{r}=0,15, \mathrm{p}=0,02)$, abuso físico materno $(\mathrm{r}=0,19, \mathrm{p}<01)$, punição paterna $(\mathrm{r}=0,21, \mathrm{p}<01)$, negligência paterna $(\mathrm{r}=0,25, \mathrm{p}=00)$ e abuso físico paterno $(\mathrm{r}=0,15, \mathrm{p}=0,03)$. O Escore Clínico Total (ECT) em relação aos Eventos Estressores (EVE) se correlacionou positivamente com a soma total dos eventos (ET) $(r=0,36, p=00)$, o impacto dos eventos (IE) $(\mathrm{r}=0,35, \mathrm{p}=00)$, eventos traumáticos no domínio social $(\mathrm{r}=0,38, \mathrm{p}=00)$, dominio pessoal $(\mathrm{r}=0,37, \mathrm{p}=00)$, dominio escolar $(\mathrm{r}=0,27, \mathrm{p}=00)$, domínio sexual $(\mathrm{r}=0,25$, $\mathrm{p}=00)$, domínio judicial $(\mathrm{r}=0,26, \mathrm{p}<01)$ e dominio familiar $(\mathrm{r}=0,24, \mathrm{p}<01)$. 
Tabela 2 - Regressão linear entre variáveis e diagnóstico clínico de externalização

\begin{tabular}{llllll}
\hline Variáveis & $\beta$ & S. E. & Exp. (Beta) & $\mathrm{t}$ & $\mathrm{p}$ \\
\hline Eventos estressores & & & & & \\
Domínio pessoal & 0,86 & 0,29 & 0,220 & 2,96 & 0,003 \\
Repertório materno & $-0,17$ & 0,08 & $-0,161$ & $-2,05$ & 0,041 \\
Repertório paterno & $-0,16$ & 0,08 & $-0,150$ & $-1,99$ & 0,048 \\
Eventos violência total & 0,18 & 0,06 & 0,206 & 2,76 & 0,006 \\
Constante & 40,02 & 4,50 & & 8,89 & 0,000 \\
\hline
\end{tabular}

R (externalização): $23.5 \%$

Para determinar as variáveis preditoras da variável independente Escore Clínico Total, realizou-se a análise de regressão linear, método Stepwise, separadamente para o diagnóstico clínico de internalização e externalização, incluindo-se as variáveis dependentes: eventos estressores (EVE) total e respectivos domínios (pessoal, escolar, social, sexual, judicial e familiar), exposição aos Eventos de Violência total e domínios (comunitário, familiar, drogas, sexual e jurídico) e estilo parental e as práticas educativas (monitoria positiva, comportamento moral, punição inconsistente, negligência, disciplina relaxada, monitoria negativa e abuso físico). Os resultados indicam que o diagnóstico clínico de externalização tem como preditores a exposição a eventos de violência (variância de 3\%,p=0,01), eventos estressores do domínio pessoal (variância de $11 \%, \mathrm{p}=00$ ) e estilos parentais materno (variância de $7 \%, \mathrm{p}=01$ ) e paternos
$(2,5 \%$ da variância, $\mathrm{p}=0,04)$. Quanto ao diagnóstico clínico de internalização, a regressão linear apontou como preditores a exposição à violência direta familiar (variância de $2 \% ; \mathrm{p}=0,01$ ), eventos estressores do dominio pessoal (variância de $11 \%, \mathrm{p}=0,00$ ) e estilos parentais paternos ( $4 \%$ da variância, $\mathrm{p}=00$ ). Considerando que o domínio pessoal contribuiu significativamente nos dois modelos de regressão linear, tanto para o diagnóstico de internalização como de externalização, realizou-se uma análise da correlação entre as variáveis (situações de violência pessoal, financeiras, aparência física, doenças, dentre outras). Verificou-se que dentre as 14 situações nesse domínio, crises nervosas ou ansiedade apresentaram uma correlação moderada com o diagnóstico clínico de internalização $(r=0,32)$ e problemas com o corpo e aparência pessoal $(\mathrm{r}=0,24)$ tiveram uma correlação fraca com o diagnóstico de externalização.

Tabela 3 - Regressão linear entre varáveis e diagnóstico clínico de internalização

\begin{tabular}{lccccc}
\hline Variáveis & B & S. E. & Exp. (Beta) & t & p \\
\hline Domínio pessoal & 1,07 & 0,23 & 0,290 & 3,91 & 0,000 \\
Repertório paterno & $-0,18$ & 0,07 & $-0,190$ & $-2,55$ & 0,011 \\
Violência familiar & 1,33 & 0,63 & 0,150 & 2,08 & 0,038 \\
Constante & 46,67 & 2,77 & & 16,82 & 0,000 \\
\hline
\end{tabular}

$\mathrm{R}^{2}$ (internalização): $17 . \%$

\section{Discussão}

Sob uma perspectiva multidimensional na compreensão dos transtornos emocionais, este estudo teve como objetivo identificar as situações individuais, familiares e contextuais associadas à manifestação de problemas de saúde mental na adolescência. Para tal, foram investigadas as características das práticas familiares de socialização e cuidado, situações traumáticas ao longo do desenvolvimento e exposição a eventos de violência em adolescentes estudantes da região metropolitana de Porto Alegre, RS.

Inicialmente, a análise geral do grupo investigado apontou algumas características importantes em relação a questões de escolaridade e características familiares. Nesse sentido, identificou-se no grupo uma alta prevalência de casos de repetência escolar, situação presente em ambos os sexos, porém com maior frequência entre adolescentes do sexo masculino. Problemas escolares, tais como repetência e evasão escolar são situações graves no processo de individuação pessoal e inserção social, acarretando consequências prejudiciais não somente para o adolescente, mas para toda uma comunidade.

Complementar ao aspecto dos problemas na esfera escolar, a análise das práticas de socialização familiares avaliadas pelos adolescentes indicou uma alta incidência de estilos parentais de risco. Isto é, quase metade do grupo de jovens descreveu a interação, tanto paterna quanto materna como constituída de práticas parentais negativas. Ou seja, no presente estudo, os escores paternos e maternos no IEP foram negativos, situações características de famílias de risco. Conforme Gomide (2006), o escore é obtido pela diferença entre 
o somatório das práticas positivas (monitoria positiva, comportamento moral) e as práticas negativas (negligência, disciplina relaxada, abuso físico, punição inconsistente, monitoria negativa). Dessa forma, neste trabalho, as práticas parentais positivas (monitoria positiva e comportamento moral) tiveram escores muito baixos, apontando o pouco envolvimento dos pais no cuidado e supervisão dos filhos, bem como a pouca preocupação parental na transmissão de valores baseados em comportamentos de honestidade e justiça. Assim, os dados identificados por Prust e Gomide (2007) acerca do menor envolvimento em práticas de comportamento moral em famílias de risco foram similares ao deste estudo. Além disso, estudos apontam que famílias de risco apresentam maior frequência de práticas parentais negativas, bem como estresse, depressão e baixo índice de habilidades sociais (Gomide \& cols., 2005). Em conjunto, esses padrões de interação familiar estão associados à manifestação de comportamento antissocial, indicando, portanto, a necessidade do trabalho e orientação familiar no nível comunitário e escolar, a fim de promover práticas parentais voltadas ao desenvolvimento de comportamentos pró-sociais (Gomide, 2006).

Eventos estressores também se constituem como vivências potencialmente associadas aos transtornos mentais. Dentre as dimensões avaliadas, tais como eventos sociais, familiares, pessoais, escolares e sexuais, algumas situações destacaram-se como mais frequentes. As dificuldades de relacionamento na dimensão familiar com os pais e irmãos, brigas com amigos na dimensão social e rompimento de namoro na dimensão pessoal foram as situações mais recorrentes percebidas como estressoras. É interessante observar que essas situações dizem respeito às próprias características da fase adolescente de maior autonomia familiar e à importância dos vínculos extrafamiliares, tornando-se prejudiciais, porém, dependendo da frequência e intensidade. Nesse sentido, quanto à gravidade ou intensidade dos eventos, as perdas de familiares e situações de abuso sexual foram aquelas de maior impacto traumático. Esses achados são similares aos identificados nas cidades de Porto Alegre e de Novo Hamburgo (Kristensen \& cols., 2004), onde se constataram que problemas do dia a dia possuem grande frequência, envolvendo questões escolares e relações interpessoais com pares e familiares. Por outro lado, os eventos isolados e com menor frequência foram considerados de maior impacto, principalmente violência sexual, situações de liberdade limitada e de privações dos adolescentes. Em conjunto, esses dados apontam que situações traumáticas de maior intensidade, como separações, mortes e eventos de violência grave, devem receber imediata atenção pelos profissionais envolvidos nos diferentes contextos de crianças e adolescentes (escola, família, comunidade) em função da associação de maior risco de sofrimento psicológico.

Outro fator que tem se destacado na etiologia das desordens emocionais é a exposição direta e indireta à violência, vitimização associada ao transtorno de estresse pós-traumático, abuso de substâncias, problemas emocionais, educacionais e de relacionamento (Avanci, Assis, Oliveira \& Pires 2009; Lynch, 2003, Margolin \& Gordis, 2004). Os adolescentes do presente trabalho apresentaram uma alta frequência de exposição à violência comunitária, roubo, assalto e armas de fogo. Especificamente, a média masculina para a exposição de eventos de violência e para a exposição direta foi maior que a feminina, o que pode ser explicado pelo maior tempo que os meninos passam nas ruas, estando, assim, mais sujeitos à exposição. De qualquer modo, tanto a exposição direta como a indireta à violência constituem-se como situações prejudiciais ao desenvolvimento de crianças e adolescentes. Nesse sentido, em um estudo nacional recente que avaliou 479 alunos num município do Rio de Janeiro (Avanci \& cols., 2009) foi identificado que a presença de violência em diferentes contextos, tais como escola, família e comunidade, associava-se a manifestações clínicas depressivas. Portanto, a implicação de experiências de violência no cotidiano de jovens se constitui como um elemento de risco grave para a saúde mental (Flannery, Singer \& Wester, 2001, McFarland \& cols., 2003, Osofsky, 1995, Rosenthal \& Wilson, 2003).

Finalmente, identificamos uma prevalência de $31 \%$ de jovens com indicativo clínico na avaliação do instrumento CBCL. Considerando o valor conservador do ponto de corte utilizado, esses achados são preocupantes. No Brasil, numa investigação recente, que incluiu uma amostra de 89 pares mães-filhos menores de 18 anos na região de Umbu, São Paulo (Bordin \& cols., 2009), utilizando-se também do instrumento CBCL, Bordin e colaboradores (2009) identificaram uma prevalência de $36,9 \%$ de crianças e adolescentes com diagnóstico clínico de internalização e externalização, ou ambos. Esse índice foi um superior ao identificado em um estudo anterior na mesma região (Paula, Duarte \& Bordin, 2007), no qual a prevalência de transtornos emocionais foi de $24,6 \%$ numa amostra de 479 crianças e adolescentes (6-17 anos). Outros estudos encontraram índices ainda menores, tal como o estudo no Rio Grande do Sul, cidade de Pelotas, no qual foi identificado um índice de 13\% na população de 10-17 anos de idade (Benvegnu \& cols., 2005). 
Certamente, diferenças na identificação da prevalência dos transtornos emocionais refletem, além de distintos delineamentos, questões contextuais dos grupos investigados. Para Paula, Duarte e Bordin (2007), o menor índice no estudo de Benvegnu e colaboradores (2005) diz respeito ao maior poder econômico do grupo estudado. No presente trabalho, uma possível interpretação da alta prevalência refere-se ao fato de que a região de nossa amostra é uma das mais violentas do estado do Rio Grande do Sul, liderando o índice de homicídios de adolescentes (UNICEF, 2009), característica em detrimento da qualidade de vida do grupo investigado.

Além do aspecto da prevalência, observou-se que do grupo clínico, 13\% dos jovens apresentavam características depressivas, com maior prevalência no grupo feminino. Entretanto, o grupo clínico de externalização foi mais freqüente, com $17 \%$, e também apresentou um maior número de adolescentes do sexo feminino. Dessa forma, observamos que adolescentes do sexo feminino apresentaram índices maiores de comportamento agressivo do que os masculinos. Recentemente, uma pesquisa longitudinal com adolescentes realizada na Holanda num período de 10 anos, utilizando igualmente $\mathrm{o}$ instrumento $\mathrm{CBCL}$ demonstrou que, para os meninos, o escore médio das escalas de Ansiedade/Depressão, Problemas Sociais, Problemas de Delinquência, Comportamento Agressivo, Internalização, Externalização e Total de Problemas diminuiu. Já para as meninas, os escores das escalas de Manifestações Somáticas, Problemas Sociais, Problemas de Pensamento, Internalização e Externalização aumentaram. Comparando os dois grupos, as meninas tiveram maiores índices de diagnóstico internalização e externalização e, além disso, obtiveram escores iguais aos meninos na subescala de Comportamento Agressivo (Tick, Ende \& Verhulst, 2008). Conforme os autores, essas mudanças necessitam de maior investigação, a fim de se estabelecer que elementos estão contribuindo para a manifestação de comportamentos agressivos no sexo feminino e especificá-los. Igualmente, o presente estudo também reforça essa posição, no sentido de precisar os contextos e situações que poderiam influenciar as trajetórias femininas.

Quanto ao interesse em identificar as associações entre as diferentes variáveis investigadas e a manifestação de problemas de saúde mental, verificamos que, apesar de significativas, as correlações categorizavam-se no nível fraco. Entretanto, valores de associação fracos e moderados, na área das Ciências Sociais, podem ser considerados, dependendo do tamanho amostral e da análise da relação identificada (Anderson, Sweeney \& Williams, 1994).
Considerando esses aspectos, as práticas parentais paternas de risco, a frequência e impacto dos eventos estressores, principalmente nas dimensões sociais e pessoais, a exposição à violência, tanto direta como indireta, e a presença de violência nos âmbitos familiar, comunitário, escolar e sexual correlacionaramse com a manifestação de diagnóstico clínico. Dentre essas situações, a análise das regressões lineares apontou que o diagnóstico clínico de externalização estava associado à exposição à violência em geral, a situações estressoras na dimensão pessoal (ansiedade, drogas, problemas com autoimagem) e aos estilos parentais negativos, tanto paternos como maternos. Por sua vez, o diagnóstico clínico de internalização associou-se à violência no âmbito familiar, a vivências de eventos traumáticos na dimensão pessoal e ao estilo paterno negativo. No presente estudo, portanto, adolescentes envolvidos com comportamento de quebrar regras e comportamento agressivo (externalização) estavam mais expostos a episódios de violência, percebiam de forma mais estressante as mudanças corporais e a própria aparência e, por último, evidenciavam interações familiares negativas com ambos os pais. Os adolescentes com diagnóstico clínico associado a retraimento, queixas somáticas, ansiedade e depressão (internalização) tiveram mais dificuldade na interação paterna, maiores problemas de violência na família e percebiam-se com mais sintomas de ansiedade. É interessante apontar que adolescentes com comportamentos agressivos apresentavam fragilidade nas relações parentais com ambos os pais, indicativo de uma rede familiar de pouco suporte. Igualmente, a maior exposição à violência nesse grupo, além de indicar a presença de um contexto violento, também aponta para um maior envolvimento do jovem com situações violentas, tais como brigas, atos violentos, contato com armas de fogo, por exemplo. Ao contrário, jovens com características depressivas indicavam maior dificuldade na dimensão familiar e presença de ansiedade. Entretanto, mantinham uma relação familiar, a materna, mais estável.

No estudo de Bordin e colaboradores (2009) sobre problemas de saúde mental e práticas punitivas de socialização, sexo feminino, idade e trabalho materno foram fatores de risco para o diagnóstico de internalização. Já o diagnóstico de externalização manifestou-se com mais frequência nas crianças e adolescentes em arranjos familiares monoparentais, nos quais nem o pai nem um guardião masculino residiam. Ainda que atuando de forma independente, tanto a variável depressão materna como a variável punição exagerada relacionaram-se ao diagnóstico externalizante. Assim sendo, também no estudo de Bordin e colaboradores (2009) foi verificada a ausência 
de relacionamento afetivo com pelo menos um dos genitores ou responsáveis no caso do diagnóstico de externalização. Da mesma forma, é importante destacar as associações identificadas por Avanci, Assis, Oliveira e Pires (2009) entre a presença de violência em diferentes contextos, escola, família e comunidade e o escore clínico na subescala do CBCL retraimento/depressão. Nesse caso, apontaram tanto a punição severa do pai quanto a violência comunitária como situações importantes pelo impacto negativo no desenvolvimento infantil, principalmente associando-se à manifestação de psicopatologia. Portanto, considerando a manifestação clínica de transtornos emocionais, verifica-se que a presença de violência, relações familiares conflituosas e práticas de socialização negativas constituem-se como os principais elementos associados à presença de distúrbios, principalmente em relação à figura paterna. Entretanto, no caso de adolescentes com características de externalização, as dificuldades familiares foram identificadas em relação a ambos os pais, apontando uma situação de fragilidade da rede de apoio imediata ao jovem.

Algumas limitações do trabalho, porém, necessitam ser apontadas, tais como a precaução em generalizar os dados para outras regiões e as questões de necessidade de maior aprofundamento das relações causais entre os fenômenos investigados. Nesse sentido, salienta-se a inclusão da avaliação de características de saúde mental familiar, variável importante associada a possível manifestação de transtornos. Todavia, os resultados contribuem para a identificação de situações importantes, associadas à saúde mental, que indicam a necessidade de desenvolvimento de ações preventivas voltadas para a saúde mental na infância e adolescência. Nesse sentido, ainda que os achados reflitam fatores de risco já apontados na literatura, considera-se essencial o desenvolvimento de pesquisas nacionais em diferentes contextos para que se possa identificar as particularidades dos diferentes grupos sociais. Dessa forma, é possível estabelecer prioridades e estratégias sensíveis às demandas de cada comunidade.

Assim, observou-se que a presença de ambientes violentos e de relações familiares conflitivas e de pouco suporte foram marcadamente os fatores associados à manifestação de sofrimento psíquico, confirmando o impacto destas situações nas trajetórias de desenvolvimento dos jovens. Além disso, se analisarmos os índices de diagnóstico clínico encontrados neste trabalho, verifica-se que a dimensão das ações preventivas deve incluir não somente a perspectiva individual do jovem, mas também os sistemas proximais como família e escola. Tais contextos configuram-se como espaços primordiais para o reconhecimento, identificação das necessidades emocionais e desenvolvimento de ações de intervenção, exigindo, portanto, em termos das políticas públicas, a implementação de serviços, a capacitação profissional nos diferentes âmbitos de inserção de crianças e adolescentes e o estabelecimento do trabalho em rede das diferentes instituições educativas e de saúde. Estas são etapas prioritárias para a prevenção e atenção das demandas dessas faixas etárias, em especial, dos aspectos de saúde mental.

\section{Referências}

Achenbach, T. M. (1991). Integrative guide for the CBCL/4-18, YSR, and TRF profiles. Burlington: University of Vermont, Department of Psyquiatry.

Anderson, D. R., Sweeney, D. J. \& Williams, T. A. (1994). Introduction to statistics: concepts and applications. St. Paul: West Publishing Company.

Assis, S. G., Avanci, J. Q., Pesce, R. P. \& Ximenes, L. F. (2009). Situação de crianças e adolescentes brasileiros em relação à saúde mental e à violência. Ciência e Saúde Coletiva, 14, 349-361.

Avanci, J., Assis, S., Oliveira. R. \& Pires, T. (2009). Quando a convivência com a violência aproxima a criança do comportamento depressivo. Ciência \& Saúde Coletiva, 14, 383-394.

Bastos, A. C. S., Urpia, A. C. M., Pinho, L. \& Almeida Filho, N. M. (1999). O impacto do ambiente familiar nos primeiros anos de vida: um estudo com adolescentes de uma invasão de Salvador, Bahia. Estudos de Psicologia, 4, 239-271.

Benetti, S. P. C., Gama, C., Vitolo, M., Silva, M. B., D’Ávila, A. \& Zavaschi, M. L. (2006). Violência comunitária, exposição às drogas ilícitas e envolvimento com a lei na adolescência. Psico (RS), 37(3), 279-286.

Benvegnu, L. A., Fassa, A. G., Facchini, L. A., Wegman, D. H. \& Dall'Agnol, M. M. (2005). Work and behavioural problems in children and adolescents. International Journal of Epidemiology, 34, 1417-1424.

Bordin, I. A., Mari, J. J. \& Caeiro, M. F. (1995). Validação da versão brasileira do "Child Behavior Checklist" (CBCL) (Inventário de Comportamentos da Infância e Adolescência): dados preliminares. Revista ABP-APAL, 17(2), 5566. 
Bordin, I. A., Duarte, C. S., Peres, C. A., Nascimento, R., Curto, B. M. \& Paula, C. S. (2009). Severe physical punishment: risk of mental health problems for poor urban children in Brazil. Bulletin of the World Health Organization, 87, 336344.

Broecker, C. \& Jou, G. I. (2007). Práticas educativas parentais: a percepção de adolescentes com e sem dependência química. Psico-USF, 12, 235-253.

Carvalho, H. C. W., Pinheiro, A. M. V., Patrick, C. J., Krueger, R. F. \& Markon, K. E. (2007). Tradução, adaptação cultural e análise de consistência interna do inventário de externalização. Avaliação psicológica, 6, 217-227.

Cicchetti, D. \& Rogosh, F. A. (2002). A developmental psychopathology perspective on adolescence. Journal of Consulting and Clinical Psychology, 70, 6-20.

Darling, N. \& Steinberg, L. (1993). Parenting style as context: an integrative model. Psychological Bulletin, 113, 487-493.

Dekovic, M. (1999). Risk and protective factors in the development of problem behavior during adolescence. Journal of Youth and Adolescence, 28(6), 293-306.

Dell'Aglio, D. D., Benetti, S., Deretti, L., Dincao, D. B. \& Leon, J. S. (2005). Eventos estressores no desenvolvimento de meninas adolescentes cumprindo medidas sócio-educativas. Paidéia, 30(15), 119-129.

Ferlin, M., Lima, J. S., Alchieri, J. C., Kristensen, C. H. \& Flores, R. Z. (2000). Desenvolvimento do inventário de eventos estressores na adolescência (IEEA). Exponha-se 2000 - Semana de Pesquisa e Iniciação Científica, São Leopoldo, RS, Brasil, 204205.

Flannery, D. J., Singer, M. I. \& Wester, K. (2001). Violence exposure, psychological trauma, and suicide risk in community sample of dangerously violent adolescents. Journal of American Academy of Child and Adolescent Psycbiatry, 40, 435-442.

Galambos, N. L., Barker E. T. \& Almeida, D. M. (2003). Parents do matter: trajectories of change in externalizing and internalizing problems in early adolescence. Child Development, 74, 578-594.

Gomide, P. I. C. (2006). Inventário de Estilos Parentais. Modelo teórico, manual de aplicação, apuração $e$ interpretação. São Paulo: Vozes.

Gomide, P. I. C., Salvo, C. G., Pinheiro, D. P. N. \& Mello, S. G. (2005). Correlação entre práticas educativas, depressão, estresse e habilidades sociais. Psico USF, 10(2), 169-178.

Kristensen, C. H., Leon, J. S., D'Incao, D. B. \& Dell'Aglio, D. D. (2004). Análise da frequência e do impacto de eventos estressores em uma amostra de adolescentes. Interação em Psicologia, $8(1), 45-55$.

Lovejoy, M. C., Weis, R., O’Hare, E. \& Rubin, E. (1999). Development and initial validation of the parent behavior inventory. Psychological Assessment, 11, 534-545.

Lynch, M. (2003). Consequences of children's exposure to community violence. Clinical Child and Family Psychology Review, 6(4), 265-274.

King, K. M. \& Chassin, L. (2008). Adolescent stressors, psychopathology, and young adult substance dependence: a prospective study. Journal of Studies on Alcohol and Drugs, 69(5), 629-638.

Margolin, G. \& Gordis, E. B. 2004). Children's violence exposure in the family and community. Current Directions in Psychological Science, 13, 152-155.

McFarland, J. M., Groff J. Y., O’Brien J. A. \& Watson K. (2003). Behaviors of children who are exposed and not exposed to intimate partner violence: an analysis of 330 black, white, and hispanic children. Pediatrics, 112, 202-207.

OMS/World Health Organization (2003). Caring for children and adolescents with mental disorders. Setting WHO directions, Geneva.

Osofsky, J. D. (1995). The effects of exposure to violence on young children. American Psychologist, 50(9), 782-788.

Paula, C. S, Duarte, C. S. \& Bordin, I. A. (2007). Prevalence of mental health problems in children and adolescents from the outskirts of São Paulo City and estimation of service need and capacity. Revista Brasileira de Psiquiatria, 29, 11-17.

Paxton, K. C., Robinson, W., Shah, S. \& Schoeny, M. E. (2004). Psychological distress for AfricanAmerican adolescent males: exposure to community violence and social support as factors. Child-Psychiatry-and-Human-Development, 34, 281 295.

Pesce, R. P., Assis, S. G., Santos, N. \& Oliveira, R. V. C. (2004). Risco e proteção: em busca de um equilíbrio promotor de resiliência. Psicologia: Teoria e Pesquisa, 20(2), 135-143.

Prust, L. W. \& Gomide, P. I. C. (2007) Relação entre comportamento moral dos pais e dos filhos 
adolescentes. Estudos de Psicologia Campinas, 24(1) 53-60.

Richters, J. E. \& Martinez, P. (1993). The NIMH community violence project: I. Children as victims of and witnesses to violence. Psychiatry, 56, 7-21.

Rosenthal, B. S. \& Wilson, W. C. (2003). Impact of exposure to community violence and sychological symptoms on college performance among students of color. Adolescence, 38, 239-249.

Rutter, M. (2009). Understanding and testing risk mechanisms for mental disorders. Journal of Child Psychology and Psychiatry, 50, 44-52.

Shirk, S., Talmi, A. \& Olds, D. (2000). A developmental psychopathology perspective on child and adolescent treatment policy. Development and Psychopathology, 12, 835-855.

Tick, N. T., Ende, J. van der. \& Verhulst, F. C. (2008). Ten-year trends in self-reported emotional and behavioral problems of Dutch adolescents. Social Psychiatry Psychiatric Epidemiology, 43, 349-355.

UNICEF (2009). Índice de homicídios na adolescência. Obtido em 20 de abril de 2010 do World Wide Web: www.unicef.org/brazil/pt/IHA.pdf.

Waaktar, T., Borge, A. I. H., Fundingsrud, H. P., Christie, H. J. \& Torgesen, S. (2004). The role of stressful life events in the development of depressive symptoms in adolescence: a longitudinal community study. Journal of Adolescence, 27(2), 153-163.

Wagner, A., Ribeiro, L. S., Arteche, A. X. \& Bornholdt, E. A. (1999). Configuração familiar e o bem-estar psicológico dos adolescentes. Psicologia Reflexão e Crítica, 12(1), 147-156.

Waiselfisz, J. (2000). Mapa da Violência II. Os jovens do Brasil. Rio de Janeiro: UNESCO.

Wathier, J. L. \& Dell'Aglio, D. D. (2007). Sintomas depressivos e eventos estressores em crianças e adolescentes no contexto de institucionalização. Revista de Psiquiatria do Rio Grande do Sul, 29(3), 305-314.

Zavaschi, M. L., Benetti, S. P. C. \& Polanczyk, G. V. (2002). Adolescents exposed to physical violence in the community: a survey in Brazilian Public Schools. Revista Panamericana de Salud Publica, 12, 327-332.

Sobre os autores:

Silvia Pereira da Cruz Benetti possui graduação em Psicologia pela Universidade Federal do Rio Grande do Sul (1980), mestrado em Educação pela Universidade Federal do Rio Grande do Sul (1986) e doutorado em Child and Family Studies - Syracuse University (1999). É professora do Curso de Mestrado em Psicologia da Universidade do Vale do Rio dos Sinos na linha de pesquisa Clínica da Infância e Adolescência.

Adriana Pizetta é estudante do curso de graduação em Psicologia pela Universidade do Vale do Rio dos Sinos e possui bolsa de iniciação científica UNIBIC/UNISINOS.

Cristian Baqui Schwartz é estudante do curso de graduação em Psicologia pela Universidade do Vale do Rio dos Sinos e possui bolsa de iniciação científica PIBIC/CNPq.

Raíssa de Azevedo Hass é estudante do curso de graduação em Psicologia pela Universidade do Vale do Rio dos Sinos e possui bolsa de iniciação científica da FAPERGS.

Vera Lúcia Melo é estudante do curso de graduação em Psicologia pela Universidade do Vale do Rio dos Sinos. 